\title{
Temperature dependent analytical model for submicron GaAs-MESFET
}

\author{
Mohamed Djouder, Arezki Benfdila, Ahcene Lakhlef \\ Microelectronics and Nanotechnology Research Group, GRMNT, FGEI, M. Mammeri University, T.O, Algeria
}

\begin{tabular}{l} 
Article Info \\
\hline Article history: \\
Received Oct 14, 2020 \\
Revised Dec 15, 2020 \\
Accepted Apr 1, 2021 \\
\hline
\end{tabular}

\section{Keywords:}

Conductance

MESFET

Submicron

Temperature

Trans-conductance

Two-dimensional

\begin{abstract}
MESFET are used in circuits of gigahertz frequencies as they are based on gallium arsenide (GaAs) having electron mobility six times higher than that of silicon. An analytical model simulating different device current-voltage characteristics, i.e., output conductance and output transconductance of a $0.3 \mu \mathrm{m}$ gate MESFET with temperature dependence is proposed. The model is validated by comparing the results of the proposed model and those of the numerical simulation. The parameter values are computed using an intrinsic MESFET of two-dimensional geometry. In this work, the distribution of different output loads for varied applied voltages is considered. Simulation results obtained under temperature variation effects for load distribution and applied driven voltage variation are considered. The RMS and average errors between the different models and GaAs MESFET simulations are calculated to evidence the proposed model accuracy. This was demonstrated by a good agreement between the proposed model and the simulation results, which are found in good agreement. The simulation results obtained under temperature variations were discussed and found to complement those obtained in the literature. This clarifies the relevance of the suggested model analytical.
\end{abstract}

This is an open access article under the CC BY-SAlicense.

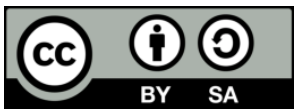

\section{Corresponding Author:}

Arezki Benfdila

Department of Electrical and Computer Engineering

Mouloud Mammeri University

Tizi-Ouzou -15000, Algeria

Email: arezki.benfdila@ummto.dz

\section{INTRODUCTION}

The essential preference of the MESFET device is based on the high mobility of electrons in the channel compared to the MOSFET device. modelling transistors I-V characteristics is an essential of any circuit design [1], when can be replaced in many applications, the most important applications today are cell phone technology, optical fiber communications systems, high performance electronic test equipment, and military transmission applications [2], [3]. MESFET-GaAs (metal semiconductor field effect transistor) made from gallium arsinide which is the basis of fast digital integrated circuits and microwave circuits and indispensable for applications operating at frequencies $>50 \mathrm{GHz}$. Important efforts have been deployed to increase the power and frequency performance of MESFET-GaAs and MESFET-GaN [4], [5]. The structures for these MESFET's are manufactured by two methods: ion-implantation and epitaxial grow [6], [7], the output characteristics of ion-implanted transistors are highly dependent on the quality of the substrate [6]-[8], for MOSFET, $\mathrm{N} 2 \mathrm{O}$ is the thin insulator deposition procedure used in the dielectric polysilicon gate of thin film transistors to achieve $\mathrm{SiO}_{2}$ layers at temperatures below $150^{\circ} \mathrm{C}$ [9].

Several analytical models have been proposed, among his known model have found the model of Larson [10], the model of Curtice and Curtice-Ettenburg model [11], [12], the disadvantage of these models 
is that they cannot simulate the dependence of the parameter $\mathrm{V}_{\mathrm{t}}$ on $\mathrm{V}_{\mathrm{DS}}$ and $\mathrm{V}_{\mathrm{GS}}$, so they are not suitable for short-channel devices [12], [13], one of the problems of the Tajima et al. [14] is that it does not take into account the simulation of $\mathrm{G}_{\mathrm{m}}$ and $\mathrm{G}_{\mathrm{d}}$, for the simulation of MESFET-DC characteristics, Rodriguez introduced an expression based on an expansion of the Curtice model depending on the parameter $\mathrm{V}_{\mathrm{t}}$ on the IDs expression [13], the adjustment of the precision of the Curtice model at the level of the linear and saturation regions is improved by the Chalmers model [15], let's look at the Dobes model [16] that we notice for $n=2$, we return to the Rodriguez model [17], the Materka-Kacprzak model [18] Seemed to be a bit more precise in the linear and saturation regions, but its accuracy degrades significantly when the device size is reduced [19], the Memon model simulates the characteristics of a MESFET-GaAs having a finite density of states at the Schottky barrier [20], by modifying the Ahmed model [17]. It has been shown that the Memon model can simulate the characteristics of the device[20], we therefore propose another new model which takes into account the interface states at the level of the Schottky barrier, which takes into account the simplicity of determining the different characteristics of the MESFET-GaAs device, such as the output transconductance, the output conductance of the MESFET device.

To improve device performances and integration, device dimensions are drastically reduced. This results in several short channel effects that affect the MESFET behavior and functionning. Reducing the size of the device conducts to a sometimes too complicated modeling of the drain current of MESFET's devices. Several models of current expressions have been presented and developed to explain the behavior of the drain current in the linear and saturation regions of the I-V characteristics, a typical explanation for the MOSFETs of Benfdila et al. [21], [22] and this is found to be the same for MESFET transistors, these effects limit the device performances by reducing the conductance $G_{m}$ and increasing transconductance $G_{d}$ when the gate length gets smaller. In this work, we describe a GaAs-MESFET modelling of the MESFET-GaAs structure, later proposed an analytical model that approaches the simulation and then we compare the results obtained with the proposed model and thus with the Curtice and Rodriguez models [11]-[13], the simulation includes fundamental equations which take into account the potential and the density of charge carriers, the poisson's equation, the transport equations, and the continuity equations [22], [23]. The impurity conditions of the trapped recombination and the various parameters that influence the static output characteristics of the MESFET device, the simulation includes the effect of temperature, to see the behavior of the device under the effect of the temperature settings on the drain current curves of the MESFET-GaAs device.

\section{DEVICE STRUCTURE AND TECHNOLOGY}

The basic structure of the MESFET-GaAs device taken in this simulation is shown in Figure 1. The deep zone is considered to be semi-insulating GaAs doped with a concentration of $\mathrm{N}_{\mathrm{a}}$, the channel is doped is an N-type with $\mathrm{N}_{\mathrm{d}}$ concentration. The implanted MESFET consists of a minimal concentration semiinsulating GaAs substrate with an implanted channel of uniform profile and maximum concentration $\mathrm{N}_{\mathrm{d}}=1 . \mathrm{e}^{16} \mathrm{~cm}^{-3}$, the thickness of the channel is $\mathrm{d}=0.12 \mu \mathrm{m}$. The $\mathrm{n}^{+}$zones implanted under the source and drain electrodes with a thickness of $0.05 \mu \mathrm{m}$ and they have a maximum concentration $\mathrm{N}_{\mathrm{d}}{ }^{+}=1 . \mathrm{e}^{18} \mathrm{~cm}^{-3}$. The gate length $\mathrm{L}_{\mathrm{g}}=0.3 \mu \mathrm{m}$, the aluminum metal was selected for the Schottky contact, the working function of the aluminum is $4.28 \mathrm{ev}$, the length of the source-gate and the length of the gate-drain $\mathrm{L}_{\mathrm{gs}}=\mathrm{L}_{\mathrm{gd}}=0.25 \mu \mathrm{m}$, the values of $\tau_{\mathrm{n}}$ and $\tau_{\mathrm{p}}$ have the value of $10^{-8}$ [24], [25], the saturation velocity $\mathrm{v}_{\mathrm{s}}=2.3 \times \mathrm{e}^{5} \mathrm{~m} / \mathrm{s}$ [25], The substrate has a thickness of $0.3 \mu \mathrm{m}$, the total thickness of the device is $0.42 \mu \mathrm{m}$ and its maximum length is $1.2 \mu \mathrm{m}$. The simulations describe the drain current, transconductance variations, the output-conductance at different bias voltage and the influence of the temperatures on drain current characteristics. These are key points discussed in this work regarding the GaAs-MESFET performances.

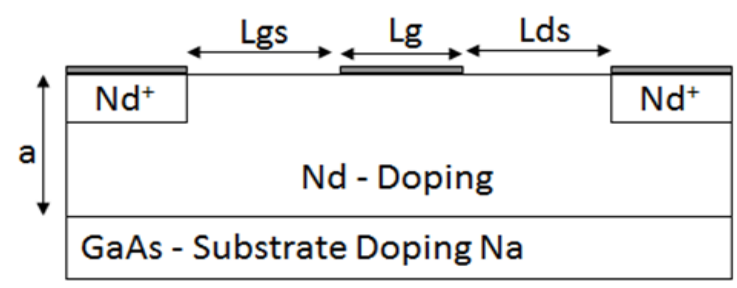

Figure 1. GaAs-MESFET device structure and geometry (after [26])

As well as the temperature is one of the essential parameters to take into account. Indeed, the temperature makes it possible to modify the performances of the device [23], [27], [28]. This work has 
developed the goal of finding a new analytical model based on our numerical simulation and compared to the two different I-V nonlinear Curtice and Rodriguezmodels for the GaAs short-channel MESFET. The Figure1 shows a two-dimensional of the GaAs-MESFET structure simulated in this work, the channel has a thickness of $0.12 \mu \mathrm{m}$ and is doped $\mathrm{N}_{\mathrm{d}}=1 . \mathrm{e}^{16} \mathrm{~cm}^{-3}$, the substrate has a thickness of $0.3 \mu \mathrm{m}$ where the shallow residual impurities $\mathrm{N}_{\mathrm{a}}=1 . \mathrm{e}^{9} \mathrm{~cm}^{-3}$ are compensated by $\mathrm{N}_{\mathrm{T}}=1 . \mathrm{e}^{15} \mathrm{~cm}^{-3}$ traps.

We have chosen the device parameters to fit the device design requirements for short channel MESFETs. These physical and geometrical parameters are typical for a short channel GaAs-MESFET device specifications and typical parameters of submicronic MESFET used in high speed digital and analog electronics are used in the proposed model are listed in the Table 1.

\begin{tabular}{|c|c|c|c|}
\hline & Simulation parameters & Values & Unites \\
\hline$N_{d}$ & Channel doping concentration & $1 . \mathrm{e}^{16}$ & $1 / \mathrm{cm}^{3}$ \\
\hline $\mathrm{N}_{\mathrm{d}}^{+}$ & Source / drain doping concentration & $1 . e^{18}$ & $1 / \mathrm{cm}^{3}$ \\
\hline$N_{a}$ & Substrate doping concentration, & $1 . \mathrm{e}^{9}$ & $1 / \mathrm{cm}^{3}$ \\
\hline$d$ & Channel Thickness & 0.12 & $u m$ \\
\hline$L_{g}$ & Metallurgical Gate length & 0.3 & $u m$ \\
\hline$L_{g s}$ & Gate-Source spacing length & 0.2 & $u m$ \\
\hline$L_{g d}$ & Gate-Drain spacing length & 0.2 & $u m$ \\
\hline$V_{b i}$ & Built-in Potential & 0.87 & $V$ \\
\hline$V_{\text {sat }}$ & Saturated value of electron drift velocity & $2.3 \mathrm{e}^{5}$ & $\mathrm{~cm} / \mathrm{s}$ \\
\hline$N_{T}$ & Substrate compensation trap & $1 . \mathrm{e}^{15}$ & $1 / \mathrm{cm}^{3}$ \\
\hline$\chi_{s}$ & Electron affinity of GaAs & 4.07 & $e V$ \\
\hline$\varphi_{m}$ & Gate metal work-function & 4.28 & $\mathrm{eV}$ \\
\hline
\end{tabular}

\subsection{Numeric simulation}

The modeling of the two-dimensional geometry of the grid short-channel MESFET including Classical semiconductor equations such as the Poisson's (1) and electric potential defined in terms of the electric field (2), the simulation involved the solution of the Poisson's, continuity equations, two nonlinear partial differential equations[31].

$$
\begin{aligned}
& \Delta^{2} \psi_{(x, y)}=-\frac{\rho_{(x, y)}}{\varepsilon_{G a A s}} \\
& \vec{E}_{(x, y)}=-\vec{\nabla} \psi_{(x, y)}
\end{aligned}
$$

The expression of the current density in relation to electric field is given by:

$$
\vec{J}_{x, y}=\sigma_{(x, y)} \vec{E}_{x, y}
$$

The MESFET was considered of type-N, where the density of current $\overrightarrow{J_{p}}$ was not taken into account during the whole simulation, which is evident for the total current density $\overrightarrow{J_{t o t}}=\overrightarrow{J_{n}}=\overrightarrow{J_{x, n}}+\overrightarrow{J_{y, n}}$, where $\overrightarrow{J_{x, n}}, \overrightarrow{J_{y, n}}$ are the density of the electron currents in the $\mathrm{x}$ and $\mathrm{y}$-directions respectively.

$$
\begin{aligned}
& \vec{J}_{x_{n}}=q \mu_{n} n \vec{E}_{x}+q D_{n} \vec{\nabla} n \\
& \vec{J}_{y_{, n}}=q \mu_{n} n \vec{E}_{y}+q D_{n} \vec{\nabla} n
\end{aligned}
$$

The electron concentration density can be written in the form $n=n_{n 0} \exp (-q V b i / K T)$, where $\mathrm{V}_{\mathrm{bi}}$ defines the built-in voltage whose value is given in Table 1 and $D_{n}$ is the diffusion of the electrons constant is given by the Einstein relationship (6), and the current density total from the channel (7).

$$
\begin{aligned}
& D_{n}=\frac{k T}{q} \mu_{n} \\
& \vec{J}_{t o t}=q\left(\mu_{n} n\left(\vec{E}_{x}+\vec{E}_{y}\right)+\left(\frac{d n}{d x}+\frac{d n}{d y}\right) D_{n}\right)
\end{aligned}
$$


The physical quantities involved in these equations are as follows:

- $\quad \mathrm{E}_{(\mathrm{x}, \mathrm{y})}$ is the electric field on $\mathrm{x}$ and $\mathrm{y}$-directions

- $\quad \Psi_{(\mathrm{x}, \mathrm{y})}$ is the total variation of the potential in the space charge zone

- $\quad \mathrm{J}_{\mathrm{x}}$ is the conduction current density in $\mathrm{x}$-direction

- $\sigma_{(\mathrm{x}, \mathrm{y})}, \rho_{(\mathrm{x}, \mathrm{y})}$ are the conductivity and the charge density in the channel respectively

Solving the Poisson equation to extract the expressions the electrostatic potential $\psi_{(\mathrm{x}, \mathrm{y})}$. Determination from (2) the components $E_{x}$ and $E_{y}$ of the electric field $E_{(x, y)}$ in the channel. Integration then determines the current $\mathrm{J}_{\mathrm{x}}$ at the source and drain electrodes, in order to establish the analytical expression of the current $I_{d}$. The current $I_{d}$ at the terminals of the device is generally calculated by integrating the total current density $\mathbf{J}_{\text {tot }}$ over an appropriate surface in each contact zone (8).

$$
I_{d}=\int_{s} J_{t o t} d s
$$

The simulation contains the implementation of the Poisson's, the current continuity equations whose flowchart is presented in appendix-A .1. The Dirichlet (fixed values) and Neumann (zero derivative values) boundary conditions for the potential are applied to the contacts and to the surface, respectively for the device.

\subsection{Classical current, conductance and transconductance models}

The classical models are investigated during decades and have been improved to match submicron MESFETs as done earlier for submicron MOSFETs. This is targeting their use in high speed digital circuits and an eventual substitution or completion of advanced CMOS technology. However, the temperature effects were not deeply studied. In this work we will introduce these effects and hence introduce a new model. The basic current equations of drain current expressions of different nonlinear MESFET of the Curtice and Rodriguez model respectively are given by the two expressions (9) and (10) respectively:

$$
\begin{aligned}
& I_{D S}=\beta\left(V_{G S}-V_{t}\right)^{2} \tanh \left(\alpha V_{D S}\right)\left(1+\lambda V_{D S}\right) \\
& I_{D S}=\beta\left(V_{G S}-V_{t}-\gamma V_{D S}\right)^{2} \tanh \left(\alpha V_{D S}\right)\left(1+\lambda V_{D S}\right)
\end{aligned}
$$

Where $\beta$ is the transconductance, $\alpha$ simulate the linear region dependence, $\lambda$ simulates the dependency of $\mathrm{I}_{\mathrm{DS}}$ in the saturation zone on $\mathrm{V}_{\mathrm{DS}}, \gamma$ simulates the threshold voltage dependency on the $\mathrm{V}_{\mathrm{DS}}, \mathrm{V}_{\mathrm{t}}$ the threshold voltage, the $\mathrm{V}_{\mathrm{t}}$ and $\Delta \mathrm{V}_{\mathrm{t}}$ are defined by [20], [32]:

$$
V_{t}=\frac{q N_{d} d^{2}}{2 \varepsilon_{s}}-\Phi_{b}
$$

Where $q$ is electron charge, $d$ is the channel thickness, $\varepsilon_{s}$ is permittivity of GaAs, $N_{d}$ the channel doping and $\Phi_{b}$ is Schottky barrier height.

$$
\Delta V_{t}=\frac{4 d}{3 L_{g}} V_{t}
$$

$\Delta \mathrm{V}_{\mathrm{t}}$ is the threshold voltage due to the submicron structure and $L_{g}$ is the gate length [33], [34].

\subsection{Conductance and transconductance equations}

The MESFET transconductance is defined by a ratio of variation of the current $I_{D S}$ by the voltage $V_{G S}(13)$ and also the conductance obtained by the variation of $I_{D S}$ by the voltage $V_{D S}$ (14) [20].

$$
\begin{gathered}
G_{d}=\frac{\partial I_{D S}}{\partial V_{D S}} \mid V_{G S}=C_{s t} \\
G_{m}=\frac{\partial I_{D S}}{\partial V_{G S}} \mid V_{D S}=C_{s t}
\end{gathered}
$$

The expression of the conductance $G_{d}$ and of the transconductance $G_{m}$, respectively of the Curtice model obtained by directing (9) according to $\mathrm{V}_{\mathrm{DS}}$ and $\mathrm{V}_{\mathrm{GS}}$ respectively are (15) and (16) respectively.

$$
G_{d}=I_{D S}\left[\frac{2 \alpha}{\sinh \left(2 \alpha V_{D S}\right)}+\frac{\lambda}{1+\gamma V_{D S}}\right]
$$


$G_{m}=\frac{2 I_{D S}}{V_{D S}-V_{t}}$

Concerning the expressions of the conductance and the transconductance of the Rodriguez model are obtained by directing the (10) following $\mathrm{V}_{\mathrm{DS}}$ and $\mathrm{V}_{\mathrm{GS}}$ respectively are (17) and (18):

$$
\begin{aligned}
& G_{d}=I_{D S}\left[\frac{-2 \gamma}{V_{G S}-V_{t}-\gamma V_{D S}}+\frac{2 \alpha}{\sinh \left(\alpha V_{D S}\right)}+\frac{\lambda}{1+\gamma V_{D S}}\right] \\
& G_{m}=\frac{2 I_{D S}}{V_{D S}-V_{t}-\gamma V_{D S}}
\end{aligned}
$$

\section{PROPOSED MODEL}

Several modeling approaches based on physical characteristics have been used to perfect and improve the electrical and thermal performance of GaAs MESFET transistors. often have a finite state interface density and the ideal device pinch. Certain physical parameters such as the barrier height, the density of the interface states, generate problems of the interface states, substrate compensation traps and non-homogeneous Schottky barrier, the model includes the voltage $\mathrm{V}_{\mathrm{t}}$ and the Schottky potential contact at the same time given in (19). In submicron GaAs-MESFETs, in order to study the submicron MESFET, we have simulated and drawn the device current-voltage (I-V) characteristics for different drain and gate voltages for temperatures ranging from $200 \mathrm{~K}$ to $600 \mathrm{~K}$ steped by $100 \mathrm{~K}$.

Assuming $\mathrm{N}_{\mathrm{d}}=1 \mathrm{e}^{16} 1 / \mathrm{cm}^{3}$ and $\mathrm{d}=0.12 \mu \mathrm{m}$ and knowing the value of $\mathrm{V}_{\mathrm{t}}$ using (11), which depends on the Schottky potential $\Phi_{b}$, the potential $\Phi_{b}$ is determined by the difference of the work-function of the metal $\Phi_{m}$ and the electron affinity $\chi_{s}$ of the GaAs semiconductor which is equal to $\Phi_{m}-\chi_{s}$, the $\Delta \mathrm{V}_{\mathrm{t}}$ is obtained by (20), which represents the shift in the threshold voltage due to the submicron structure of the MESFET whose gate length $\mathrm{L}_{\mathrm{g}}=0.3 \mu \mathrm{m}$ for the devices, whose parameters are and represented in Table 1 . The table shows that the device having $\mathrm{L}_{\mathrm{g}}=0.3 \mu \mathrm{m}$ it is with this amplitude that the transistor is classified as submicron, increasing the channel thickness $\mathrm{d}$, the performance of the component deteriorates. The basic current equation used in this study are given by the following current-voltage expression:

$$
I_{D S}=\beta \frac{T}{T_{0}}(A)^{3} \tanh \left(\alpha V_{D S}\right)\left(1+\lambda V_{D S}\right)
$$

Where $\beta$ simulate the transconductance, $\lambda$ simulates the dependence of $\mathrm{I}_{\mathrm{DS}}$ on $\mathrm{V}_{\mathrm{DS}}$ in the saturation zone, $\mathrm{V}_{\mathrm{t}}$ the threshold voltage, $\alpha$ simulate the dependency of a linear zone, $\gamma$ simulates the dependence of threshold voltage on $\mathrm{V}_{\mathrm{DS}}, \mathrm{T}_{0}$ represents the ambient temperature, $\mathrm{T}$ the temperature desired.

The expressions of the output conductance $G_{d}$ and the output transconductance $G_{m}$ in small-signal of the proposed model are deduced therefrom the expression of the current $I_{D S}$ given in (19), with regard to $V_{D S}$ and $\mathrm{V}_{\mathrm{GS}}$ respectively are given by:

$$
\begin{aligned}
& G_{d}=3 \beta \frac{T}{T_{0}} A^{2}[-\lambda \beta-C] D E+\alpha \beta F A^{3} E+\lambda \beta A^{3} E \\
& G m=3 \beta \frac{T}{T_{0}} A^{2}\left[\frac{C}{\gamma}\right] D E
\end{aligned}
$$

The indices A, B, C, D, E, F are used for the simplification of the proposed model whose associated quantities for each index are given by:

$$
\begin{aligned}
& A=\left(1-\lambda V_{D S}\right) B \\
& B=\sqrt{\left(V_{G S}-V_{t}-\Delta V_{t}-\gamma V_{D S}\right)} \\
& C=\gamma \frac{\left(1-V_{D S}\right)}{2 \beta} \\
& D=\tanh \left(\alpha V_{D S}\right) \\
& E=1+\lambda V_{D S} \\
& F=1-\tanh ^{2}\left(\alpha V_{D S}\right)
\end{aligned}
$$




\section{RESULTS AND DISCUSSIONS}

\subsection{Device DC characteristics}

The simulations are performed on the GaAs-MESFET considered in the structure of Figure 1 and the curves obtained underline the performance of devices. The $I-V$ characteristics $I D=f\left(V_{D S}, V_{G S}\right)$ for a device containing variations of the different physical and geometrical parameters of the proposed structure are presented in Figure 2.

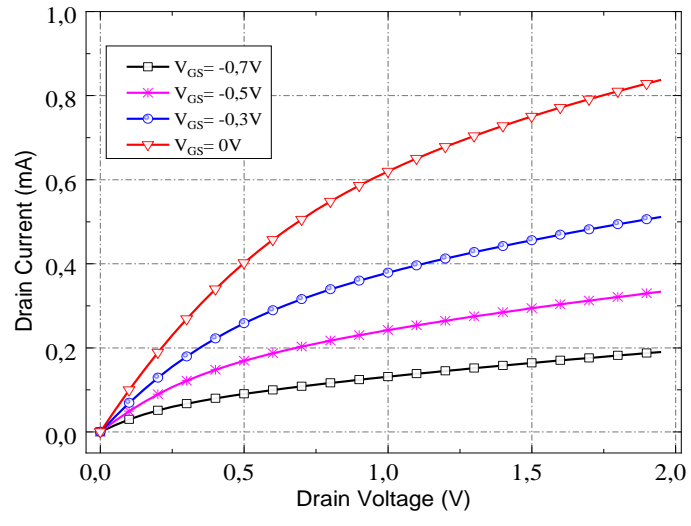

(a)

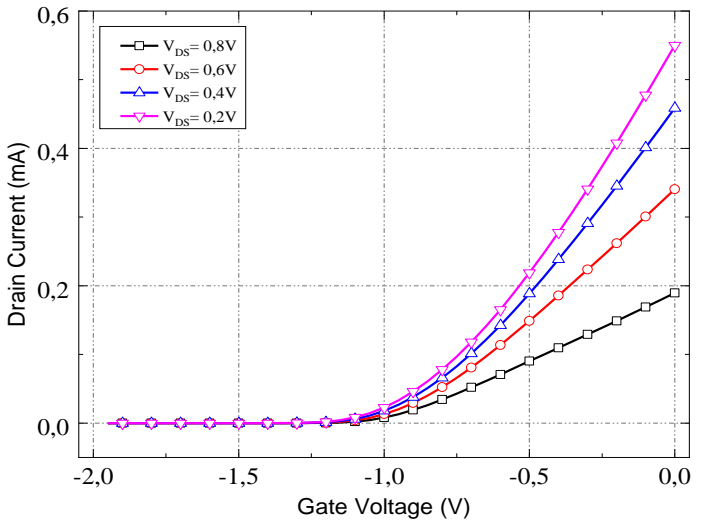

(b)

Figure 2. Characteristics of the GaAs-MESFET at room temperature obtained by simulation,(a) $\mathrm{I}_{\mathrm{DS}}-\mathrm{V}_{\mathrm{DS}}$ charactéristics with a variety of values $\mathrm{V}_{\mathrm{GS}}$, the drain voltage from 0 to $2 \mathrm{~V}$ by step $0,05 \mathrm{~V}$,

(b) $\mathrm{I}_{\mathrm{DS}}-\mathrm{V}_{\mathrm{GS}}$ charactéristics at different values of $\mathrm{V}_{\mathrm{DS}}$

The significant dependence of the device on the temperature shown in Figure 3 and examines the influence of the temperature parameter on the component's activity, is clearly visible on the transfer characteristics $I-V=f(T)$ as it is indicated for $300 \mathrm{~K}$ the current $I_{D S}=0.9 \mathrm{~mA}$ for $V_{G S}=0 \mathrm{~V}$, on the other hand for $400 \mathrm{~K}$ the current has increased about $0.05 \mathrm{~mA}$, the temperature changes the curved shapes in the linear region.

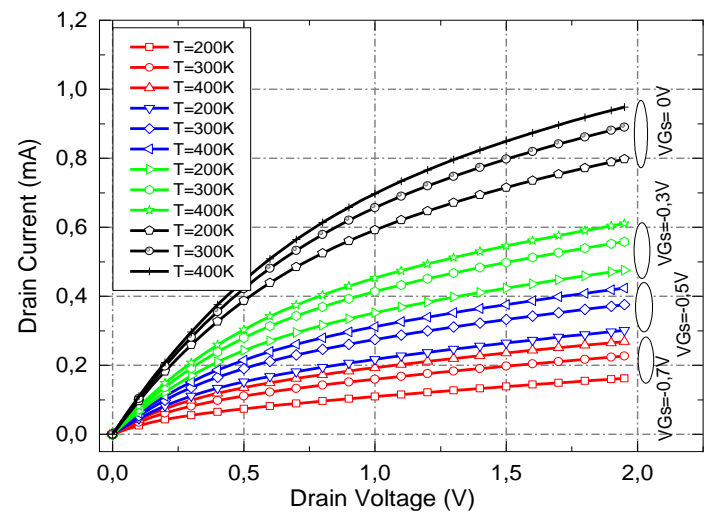

(a)

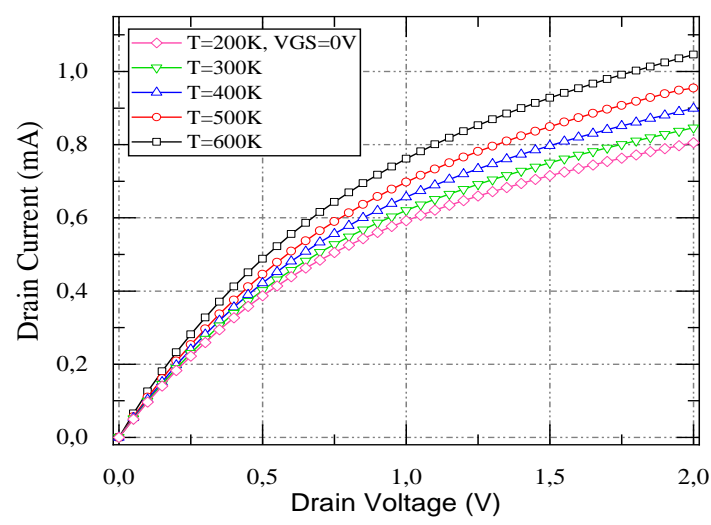

(b)

Figure 3. Characteristics $I_{D S}-V_{D S}$ obtained by simulation with a variety of values of temperature, (a) Different values of bias $\mathrm{V}_{\mathrm{GS}}$ polarization, (b) Fixed $\mathrm{V}_{\mathrm{GS}}$ to $0 \mathrm{~V}$

The Figures $4(\mathrm{a}$ and $\mathrm{b})$ show the characteristics $\mathrm{I}_{\mathrm{DS}}=\mathrm{f}\left(\mathrm{V}_{\mathrm{DS}}, \mathrm{V}_{\mathrm{GS}}\right)$ for the different gate and drain voltage values, respectively, the results obtained by the simulation of the proposed 2D structure are compared with the proposed model, the results of the model are similar and reasonable by comparing them with Rodriguez, Curtice and the simulation, which brings us back to the validation of our model.

The comparison shows a low average error between the simulation and the proposed model, however, the precision obtained is acceptable in the manufacture of electronic devices, the average errors of the different curves illustrated in Figures 4( $a$ and $b)$ are represented in the Table 2. 


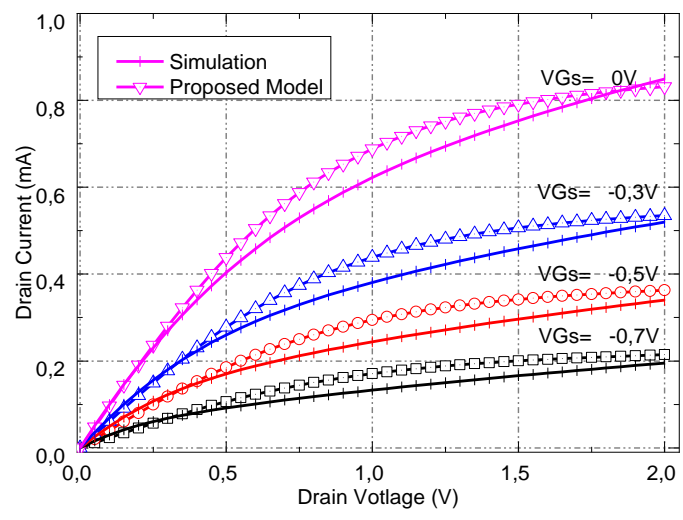

(a)

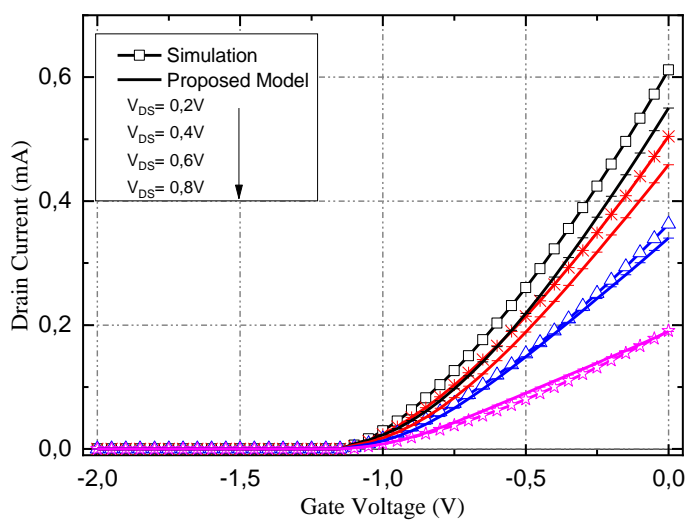

(b)

Figure 4. Comparison of features of GaAs-MESFET between the simulation and the model proposed, (a) Comparison of the $\mathrm{I}_{\mathrm{DS}}-\mathrm{V}_{\mathrm{DS}}$ obtained by simulation and proposed model with a variety of values $\mathrm{V}_{\mathrm{GS}}$,

(b) Comparison of the $\mathrm{I}_{\mathrm{DS}}-\mathrm{V}_{\mathrm{GS}}$ obtained by simulation and proposed model with at different values of $\mathrm{V}_{\mathrm{DS}}$

Table 2. Comparison of the RMS errors and average error of GaAs-MESFETs

\begin{tabular}{ccccccc}
\hline \multirow{2}{*}{ Different $\mathrm{V}_{\mathrm{GS}}$} & \multicolumn{2}{c}{$\begin{array}{c}\text { RMS errors at different } \mathrm{V}_{\mathrm{GS}} \text { results of } \\
\text { Figure 4(a) }\end{array}$} & \multicolumn{2}{c}{ Different $\mathrm{V}_{\mathrm{DS}}$} & \multicolumn{2}{c}{$\begin{array}{c}\text { RMS errors at different } \mathrm{V}_{\mathrm{DS}} \text { results } \\
\text { of Figure 4(b) }\end{array}$} \\
\cline { 2 - 3 } & Simulation & Proposed model & & Simulation & Proposed model \\
\hline $\mathrm{V}_{\mathrm{GS}}=0 \mathrm{~V}$ & 0.13 & 0,16 & & $\mathrm{~V}_{\mathrm{DS}}=0.2 \mathrm{~V}$ & 0.21 & 0.24 \\
$\mathrm{~V}_{\mathrm{GS}}=-0.3 \mathrm{~V}$ & 0.24 & 0.27 & & $\mathrm{~V}_{\mathrm{DS}}=0.4 \mathrm{~V}$ & 0.18 & 0.20 \\
$\mathrm{~V}_{\mathrm{GS}}=-0.5 \mathrm{~V}$ & 0.37 & 0.41 & & $\mathrm{~V}_{\mathrm{DS}}=0.6 \mathrm{~V}$ & 0.14 & 0.14 \\
$\mathrm{~V}_{\mathrm{GS}}=-0.7 \mathrm{~V}$ & 0.61 & 0.64 & & $\mathrm{~V}_{\mathrm{DS}}=0.8 \mathrm{~V}$ & 0.08 & 0.07 \\
Average Error & 0.34 & 0.37 & Average Error & 0.15 & 0.16 \\
\hline
\end{tabular}

The RMS and average error values were calculated (28) for the different bias voltages $\mathrm{V}_{\mathrm{GS}}$, $\mathrm{V}_{\mathrm{DS}}$ respectively for the models considered as indicated in Table 2 respectively, the precision of the model is evaluated by referring to the RMS error values as a function of both $\mathrm{V}_{\mathrm{GS}}$ and $\mathrm{V}_{\mathrm{DS}}$, observing that the mean error of the model predicted with a very small deviation from the mean error of the results obtained by simulation in the two cases. To calculate the relative error where the error in percentage in this study of the different curves obtained during this work using:

$$
R M S(\text { Error })=\sqrt{\frac{\sum_{i=1}^{N}\left(\widehat{y}_{l}-y_{i}\right)^{2}}{N}} * 100 \%
$$

The RMSerror values as shown in Table 3 for Figures 5(a and b), the RMS error values for the various $\mathrm{V}_{\mathrm{GS}}$ values obtained by the simulation lower than that obtained by the Curtice and Rodriguez model respectively and also note the Average error values are also lower. In Figures 6(a and b), show that the suggested model is exhaustive and that it can simulate the output characteristics of the GaAs MESFET under different grid conditions, in comparison with already existing models.

Table 3. Comparison of the RMS errors and average error of GaAs-MESFETs with a Curtice and Rodriguez models

\begin{tabular}{ccccc}
\hline \multirow{2}{*}{ Different $\mathrm{V}_{\mathrm{GS}}$} & \multicolumn{4}{c}{ RMS errors for different $\mathrm{V}_{\mathrm{GS}}$ compared with simulation and proposed model } \\
\cline { 2 - 5 } & Rodriguez Model & CurticeModel & By Simulation & Proposed Model \\
\hline $\mathrm{V}_{\mathrm{GS}}=0 \mathrm{~V}$ & 0.36 & 0.36 & 0.13 & 0.16 \\
$\mathrm{~V}_{\mathrm{GS}}=-0.3 \mathrm{~V}$ & 0.37 & 0.38 & 0.24 & 0.27 \\
$\mathrm{~V}_{\mathrm{GS}}=-0.5 \mathrm{~V}$ & 0.60 & 0.59 & 0.37 & 0.41 \\
$\mathrm{~V}_{\mathrm{GS}}=-0.7 \mathrm{~V}$ & 0.60 & 0.62 & 0.61 & 0.64 \\
Average Error & 0.48 & 0.49 & 0.34 & 0.37 \\
\hline
\end{tabular}




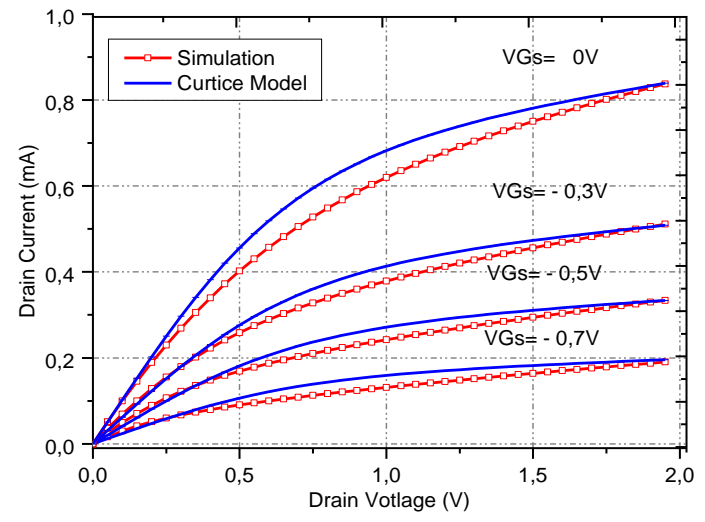

(a)

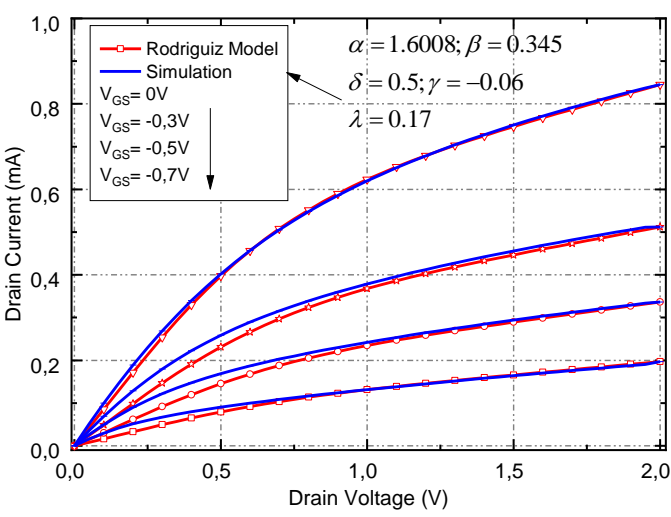

(b)

Figure 5. Comparison of MESFET characteristics, (a) $I_{D S}-V_{D S}$ comparison between the simulation and Curtice model, (b) $\mathrm{I}_{\mathrm{DS}}-\mathrm{V}_{\mathrm{DS}}$ comparison between the simulation and Rodriguez model

The RMS error values of Figures 6(a and b) are also presented in the same table, the results obtained by the suggested model are lower than those of the two models Curtice and Rodriguez respectively and also for the values Average error, including the results obtained by the proposed model, are lower than those of the two models, respectively Curtice and Rodriguez, as well as for the average error values.

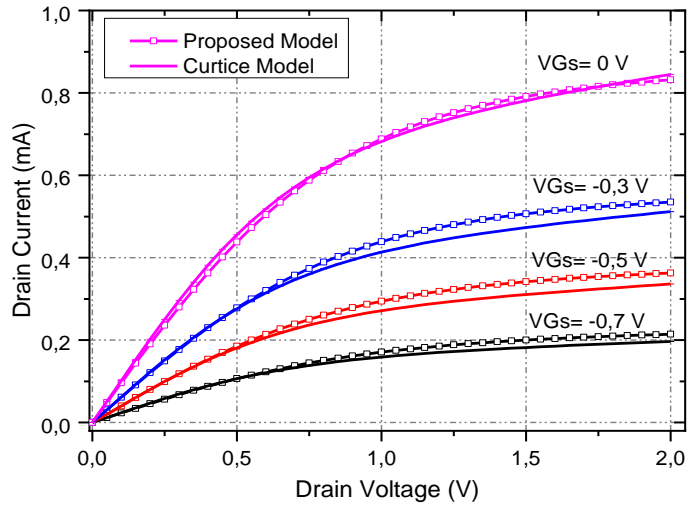

(a)

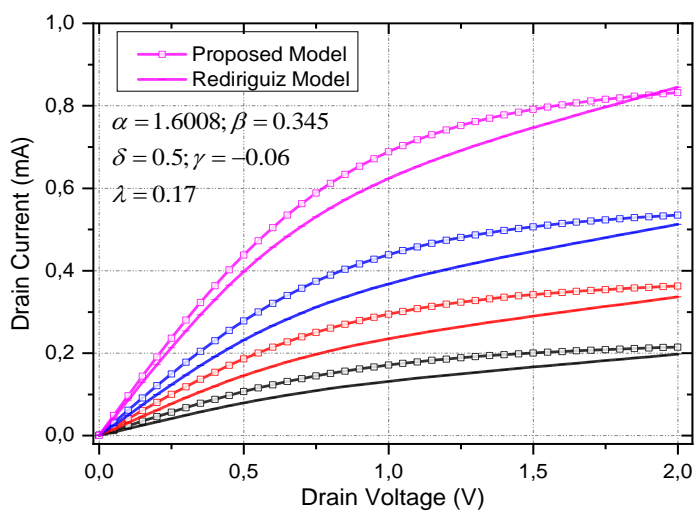

(b)

Figure 6. Comparison the $\mathrm{I}_{\mathrm{DS}}-\mathrm{V}_{\mathrm{DS}}$ characteristics of MESFET, (a) Comparison between the proposed model and Curtice model, (b) Comparison between the proposed model and Rodriguez model

The parameters defined during the simulation of the proposed model are the following: $\alpha=1.2$; $\beta=0.665 ; \gamma=-0.02 ; \lambda=0.001$, in contrast to models Curtice and Rodriguez are: $\alpha=1.6008 ; \beta=0.345$; $\gamma=-0.006 ; \lambda=0.17$.

\subsection{Conductance and output transconductance}

The values of $G_{d}$ and $G_{m}$ for the device are presented in Figures 7(a and b), 8(a and b) for all $V_{D S}$ and $\mathrm{V}_{\mathrm{GS}}$ apply and different temperatures, comparing the simulation of the device and the proposed model, shows a good agreement that confirms the validity of the proposed model, we may assume that it is possible to simulate a larger range of MESFET's with greater precision with the proposed model.

Figures $8(\mathrm{a}$ and $\mathrm{b})$, show the output conductance and transconductance of the drain, respectively as a function of $V_{D S}$ for the values applied to the gate and as a function of $V_{G S}$ for the voltages imposed on the drain, respectively in comparison between the proposed model and the simulation of the device, let's look model [17] does not take into account the effects of gate polarization $V_{D S}$, on output conductance $G_{d}$, device 
operation in the saturation zone were avoid in Islam et al. [35] made a modification to this model, showed that the effect of bias voltages $\left(\mathrm{V}_{\mathrm{DS}}-\mathrm{V}_{\mathrm{GS}}\right)$ on the output conductance of the built-in device. The proposed model shows an excellent precision concerning the output conductance presented in Figure 8(a), the transconductance represented in Figure 8(b) in comparison between the simulation and the proposed model show an almost similar, which brings us back to justify and validate this model to easily determine the different characteristics of the MESFET-GaAs device.

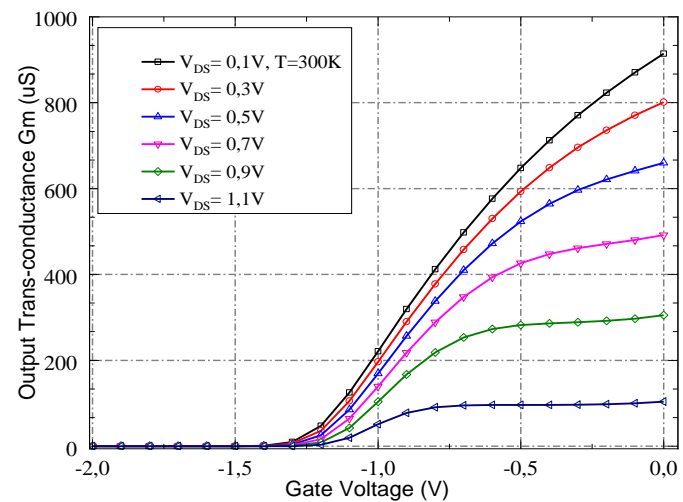

(a)

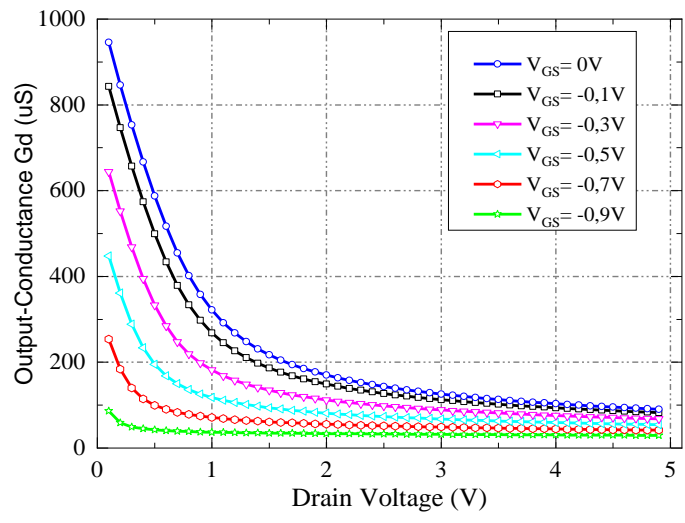

(b)

Figure 7.The transconductance, the conductance at ambient temperature obtained by simulation of MESFETGaAs, (a) The output-transconductance $G_{m}\left(V_{G S}\right)$ at different $V_{D S}$, (b) The output-conductance $G_{d}\left(V_{D S}\right)$ at different $V_{\mathrm{GS}}$

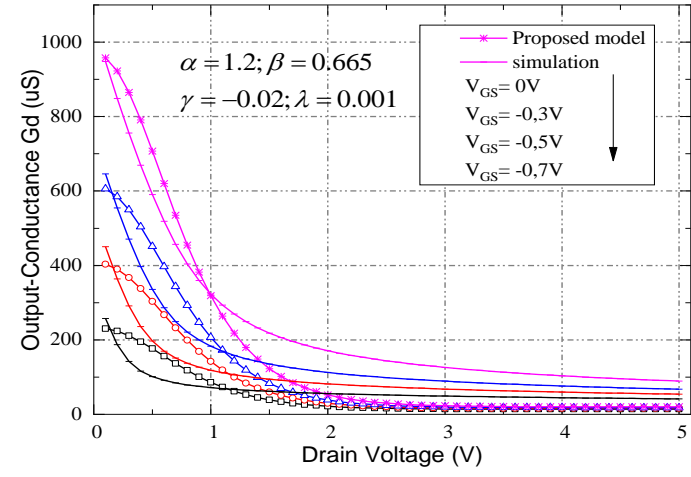

(a)

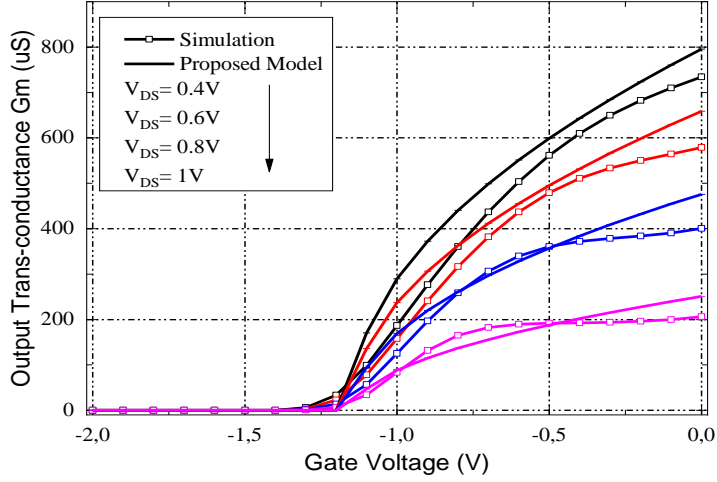

(b)

Figure 8. Characteristics of the conductance and the transconductance of the GaAs-MESFET device for the ambient temperature in comparison between the model suggested and the model of simulation, (a) The output-conductance $\mathrm{G}_{\mathrm{d}}\left(\mathrm{V}_{\mathrm{DS}}\right)$ at different gate voltage, (b) The output-transconductance $\mathrm{G}_{\mathrm{m}}\left(\mathrm{V}_{\mathrm{GS}}\right)$ at different drain voltage

\section{CONCLUSION}

All of this work contributes to the implementation of a simulation taking into account the influence of important parameters when there may be an influence on the characteristics of the MESFET GaAs transistor. Our study has been processed and written to determine the output IV, conductance and transconductance characteristics of the GaAs MESFET, to simulate the I-V features of the GaAs MESFET device, a completely new model has been developed for a short submicron channel. The results of the model established show a correlation well to our simulation results, the efficiency of this model compared with the models available in the literature, the RMS error and the average error were calculated and showing reasonable accuracy of the model, the model could be a useful tool for the design and characterization of 
integrated circuits, it has also been shown that the new proposal is a valid model capable of obtaining all current-voltage GaAs-MESFET, conductance and transconductance characteristics, the effect of temperature on the output characteristics of the device was discussed. it has been shown that the temperature is one of the essential parameters to be taken into account. In fact, the temperature makes it possible to modify the performance of the components. As indicated, this type of device can operate at high temperatures appears in all the figures obtained as a function of the temperatures. The effect of these parameters on performance has been observed and presented, its results compare favorably with a submicron GaAs MESFET studied by several authors.

\section{APPENDIX -A}

A-1; A MATLAB script is used in the modeling of the device which takes into account the various geometric and physical parameters of which the main program flowchart is illustrated in Figure A-1. The simulation code is developed using the MATLAB software which consists in modeling and simulating the device by the finite element method for the prediction of the characteristics of the MESFET sub-micron transistor, the geometry of which we have taken in Figure 1 with the parameters shown in the Table 1. The simulation program can be used on the one hand for the design of field effect devices, the parametric configuration can be carried out to allow on the other hand, by calculating the various curves of the device, the simulation also takes take into account the effects of temperature variation as an important parameter that should not be neglected as long as the temperature changes and affects the correct operation of the device, the proposed model of the GaAs MESFET is developed to obtain a comparison between the simulation and the best-known Curtice and Rodriguez models, this model is implanted on the MATLAB, the model is presented in an exhaustive way where the current, transconductance and conductance have been presented.
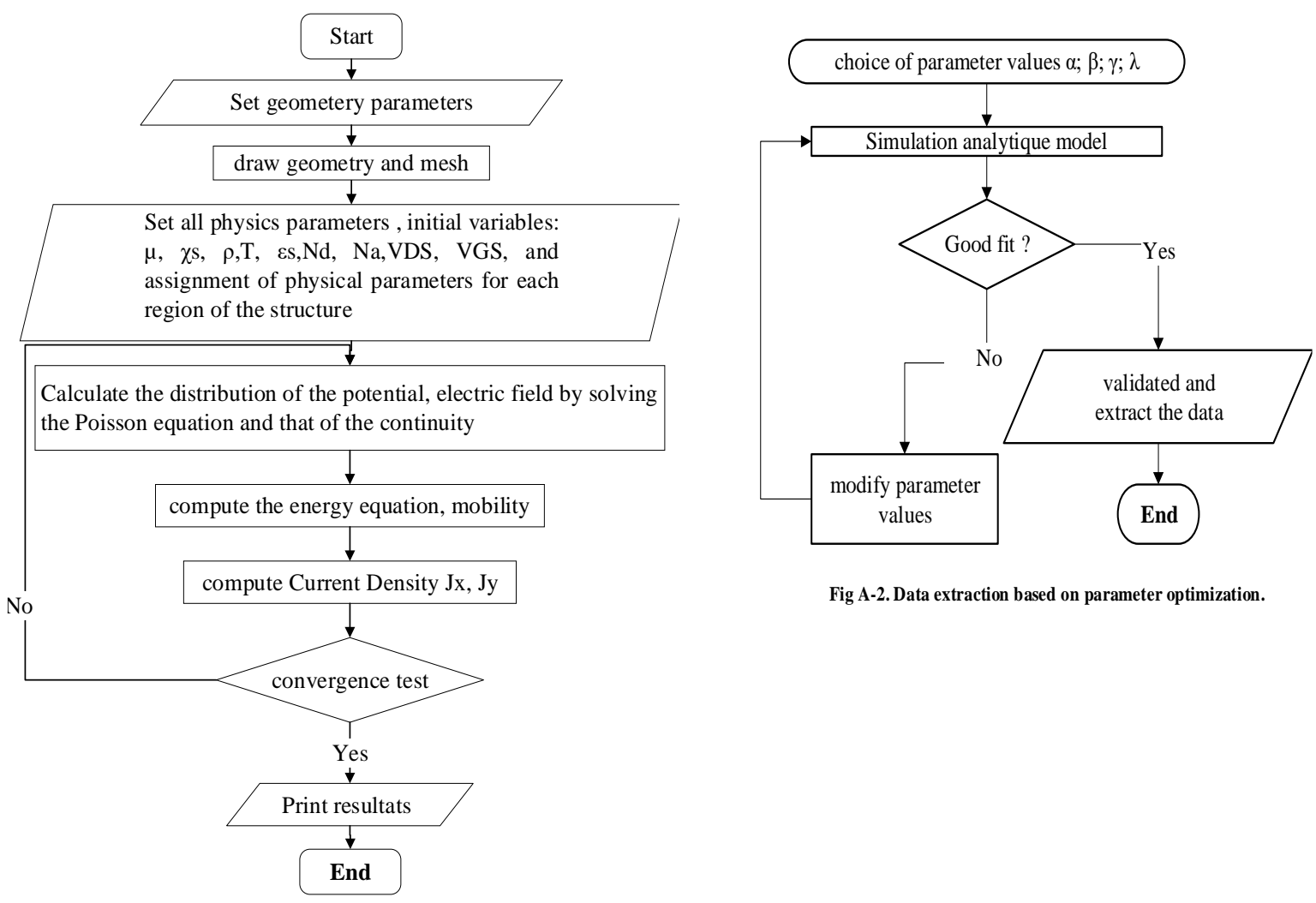

Fig A-2. Data extraction based on parameter optimization.

Fig. A-1. Main flowchart of the computer program implemented under Matlab to simulate the differents characteristics

A-2; The iterative process of optimizing the new analytical model proposed, including the optimal parameters corresponding to the results of our simulation and those of the Curtice and Rodriguez models were defined after the comparison of the data and the validation of the proposed model. The simulation steps model are: 1) definition of values: $\left.\alpha, \beta, \gamma, \lambda, a, L_{g} ; 2\right)$ calculate from $\left.V_{t}, \Delta V_{t}, \Phi_{b} ; 3\right)$ writing of the current equation (IDS), conductance $\left(\mathrm{G}_{\mathrm{d}}\right)$ and transconductance $\left(\mathrm{G}_{\mathrm{m}}\right)$ equations; 4) test program; 5) comparison and validation. 


\section{REFERENCES}

[1] S. Valiollahi, and G. Ardeshir, "A Simple General-purpose I-V Model for All Operating Modes of Deep Submicron MOSFETs," IJE TRANSACTIONS B: Applicationsvol. 31, no. 2, pp.270-277, February 2018.

[2] A. Houda, and Z. Mourad, "Electromagnetic Modeling of Active Circuit using Wave Concept Iterative Process,"Bulletin of Electrical Engineering and Informatics, vol. 5, no. 2, pp.187-193, June 2016, doi: 10.11591/eei.v5i2.528.

[3] Albert G. Baca, and I.H. Carol Ashby, "Fabrication of GaAs devices," United Kingdom; The Institution of Engineering and Technology, September 2005, pp. 350.

[4] N. Syakirah, et al., "High gain over an octave bandwidth class-F RF power amplifier design using 10W GaNHEM," Bulletin of Electrical Engineering and Informatics (BEEI), vol. 9, no. 5, pp.1899-1906, October 2020.

[5] N. Babapour, and J. Javidan, "Design of a Class F Power Amplifier With 60\% Efficiency at $1800 \mathrm{MHz}$ Frequency," Bulletin of Electrical Engineering and Informatics (BEEI), vol. 4, no. 4, pp.314-319, December 2015, doi: 10.11591/eei.v9i5.2226.

[6] M. Djouder, A. Benfdila and A. Lakhlef, "Effect of Varying Temperature on GaAs-MESFET Electrical Parameters," ICTACT Journal on Microelectronics, vol.4, no.4, pp.705-709, January 2019, doi: 10.21917/ijme.2019.01123.

[7] S Jit, S Morarka, S Mishra, "A 2-D Model for the Potential Distribution and Threshold Voltage of Fully Depleted Short-Channel Ion-Implanted Silicon MESFET's," Journal of Semiconductor Technology and Science, vol.05, no.03, pp.173-180, September 2005.

[8] A. K. Shestakov, K. S. Zhuravlev, V. S. Arykov and V. A. Kagadei, "Doping profile influence on characteristics of ion-implanted gaas field effect transistor with the schottky barrier," 2010 IEEE Region 8 International Conference on Computational Technologies in Electrical and Electronics Engineering (SIBIRCON), Irkutsk, Russia, 2010, pp. 584-588, doi: 10.1109/SIBIRCON.2010.5555371, doi: 10.1109/SIBIRCON.2010.5555371.

[9] S. Rastani, and H. Babaib, "Process Optimization of Deposition Conditions for Low Temperature Thin Film Insulators used in Thin Film Transistors Displays, "IJE TRANSACTIONS B: Applicationsvol. 31, no. 5, pp: 712-718, May 2018.

[10] L. E. Larson, "An improved GaAs MESFET equivalent circuit model for analog integrated circuit applications," in IEEE Journal of Solid-State Circuits, vol. 22, no. 4, pp. 567-574, Aug. 1987, doi: 10.1109/JSSC.1987.1052774.

[11] W. R. Curtice and M. Ettenberg, "A Nonlinear GaAs FET Model for Use in the Design of Output Circuits for Power Amplifiers," in IEEE Transactions on Microwave Theory and Techniques, vol. 33, no. 12, pp. 1383-1394, Dec. 1985, doi: 10.1109/TMTT.1985.1133229

[12] W. R. Curtice, "A MESFET Model for Use in the Design of GaAs Integrated Circuits," in IEEE Transactions on Microwave Theory and Techniques, vol. 28, no. 5, pp. 448-456, May 1980, doi: 10.1109/TMTT.1980.1130099.

[13] J. Rodriguez-Tellez, and P. J. England, "Five-Parameter DC GaAs MESFET Model for Nonlinear Circuit Design," IEE Proceedings G (Circuits, Devices and Systems), vol. 139, no. 03, pp.325-332, June 1992, doi: 10.1049/ip-g2.1992.0053.

[14] Y. Tajima, B. Wrona and K. Mishima, "GaAs FET large-signal model and its application to circuit designs," in IEEE Transactions on Electron Devices, vol. 28, no. 2, pp. 171-175, Feb. 1981, doi: 10.1109/T-ED.1981.20306.

[15] I. Angelov, L. Bengtsson and M. Garcia, "Extensions of the Chalmers nonlinear HEMT and MESFET model," in IEEE Transactions on Microwave Theory and Techniques, vol. 44, no. 10, pp. 1664-1674, Oct. 1996, doi: 10.1109/22.538957.

[16] J. Dobes, and L. Pospisil, "Enhancing the Accuracy of Microwave Element Models by Artificial Neural Networks," Radioengineering-Prague, vol. 13, pp.7-12, September 2004.

[17] M. Ahmed, M. H. Ahmed, and P. H. Ladbrooke, "An Improved DC Model for Circuit Analysis Programs for Submicron GaAs MESFET's," IEEE Transactions on Electron Devices, vol.44, no.3, pp.360-363, March 1997, doi: $10.1109 / 16.556144$.

[18] T. Kacprzak, and A. Materka, "Compact DC model of GaAs FETs for large-signal computer calculation," IEEE Journal of Solid-State Circuits, vol. 18, no. 02, pp.211-213, April 1983, doi: 10.1109/JSSC.1983.1051924.

[19] S. Tripathi, and S. Jit, "A two-dimensional (2D) analytical model for the potential distribution and threshold voltage of short-channel ion-implanted GaAs MESFETs under dark and illuminated conditions," JSTS:Journal of Semiconductor Technology and Science, vol. 11, no. 1, pp.40-50, March 2011, doi: 10.5573/JSTS.2011.11.1.040.

[20] N.M.Memon, M.M.Ahmed, F.Rehman, "A Comprehensive Four Parameters I-V Model for GaAs MESFET output characteristics,"Solid-State Electronics, vol. 51, no. 3, pp.511-516, March 2007, doi: 10.1016/j.sse.2006.12.011.

[21] A. Benfdila, and F. Balestra, "On the drain current saturation in short channel MOSFETs,"Microelectronics journal, vol.37, no.7, pp.635-641, July 2006, doi: 10.1016/j.mejo.2005.09.020.

[22] A. Lakhlef, et al., "Unified current modelling in low-dimensional MOSFETs, "International Journal of Numerical Modelling: Electronic Networks, Devices and Fields, vol. 27, no. 05, pp.908-914, February 2014, doi: 10.1002/jnm.1976.

[23] S. Bose, et al., "Model for optically biased short-channel GaAs MESFET," Microwave Opt. Technol. Lett, vol. 32, no. 2, pp.138-142, January 2002, doi: 10.1002/mop.10113.

[24] W. R. Curtice, and Yong-Hoon Yun, "A temperature model for the GaAs MESFET," IEEE Transactions on Electron Devices, vol. 28, no. 8, pp.954-962, Aug 1981,doi: 10.1109/T-ED.1981.20466.

[25] J.C. Manifacier, and R. Ardebili, "Backgating effect in III-V MESFET's: A physical model, "Solid-State Electronics, Science Direct, vol. 91, pp.13-18, January 2014, doi: 10.1016/j.sse.2013.09.005.

[26] Y. A. Khalaf, "Systematic Optimization Technique for MESFET Modeling, "Virginia Tech Electronic Theses and Dissertations Doctoral dissertation, July 2000. 
[27] K. Yamasaki, and M. Hirayama, "Determination of effective saturation velocity in $\mathrm{n}+$ self-aligned GaAs MESFETs with submicrometer gate lengths," in IEEE Trans. Electron Devices, vol. 33, no. 11, pp.1652-1658, November 1986,doi: 10.1109/T-ED.1986.22724.

[28] Kabra, et al., "Temperature Dependent Analytical Model of Sub-micron GaN MESFETs for Microwave Frequency Applications, "Solid-state electronics, vol. 52, no. 01, pp.25-30, January 2008, doi: 10.1016/j.sse.2007.06.010.

[29] M.S. Shur, et al., "Unified GaAs MESFT Model for Circuit Simulations," International Journal of High-Speed Electronics and systems, vol. 03, no. 02, pp.201-233, May 1992, doi: 10.1142/S0129156492000084.

[30] Y. Beddiafi, et al.,"Numerical Simulation of Radiation Damage on The Device Performance of GaAs MESFETs," Journal of New Technology and Materials JNTM, vol. 4, no.01, pp. 68-72, 2014.

[31] S. Chin and C. Wu, "A new I-V model for short gate-length MESFET's," in IEEE Transactions on Electron Devices, vol. 40, no. 4, pp. 712-720, April 1993, doi: 10.1109/16.202782.

[32] M. K. Verma, and B. B. Pal, "Analysis of Buried Gate MESFET under Dark and Illumination," IEEE Transactions on Electron Devices, vol. 48, no. 9, pp.2138-2142, September 2001, doi: 10.1109/16.944207.

[33] M.S. Islam, and M.M. Zaman, "A seven-parameter nonlinear I-V characteristics model for sub-lm range GaAs MESFETs," Solid-State Electronics, vol. 48, no. 7, pp.1111-1117, July 2004, doi: 10.1016/j.sse.2004.01.007.

[34] P.J. McNally, and B. Daniels, "Compact DC model for submicron GaAs MESFETsincluding gate-source modulation effects," Microelectronics Journal, vol. 32, no. 03, pp.249-251, March 2001, doi: 10.1016/S00262692(00)00103-8.

[35] M. S. Islam, M. Islam, M. R. Hasan and S. M. N. Islam, "An improved nonlinear DC I-V characteristics model for nanometer range GaAs MESFETs," TENCON 2009-2009 IEEE Region 10 Conference, Singapore, 2009, pp. 1-5, doi: 10.1109/TENCON.2009.5395879, doi: 10.1109/TENCON.2009.5395879.

\section{BIOGRAPHIES OF AUTHORS}
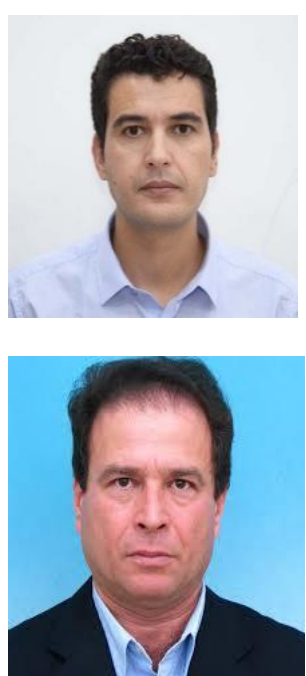

Mohamed Djouder received his Automatics Engineering degree from Mouloud Mammeri University, Tizi-Ouzou, Algeria in 2012. And he is receved a Master degree on Electrical Engineering in 2015. Currently he is a PhD student at Mouloud Mammeri University of TiziOuzou, Algeria. His research interests include modeling of microelectronics and nanotechnology devices based on semiconductors.

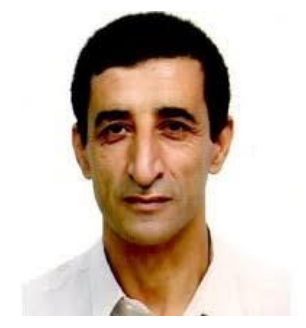

Arezki Benfdila He received from the National Institute of Electricity and Electronics, INELEC, Boumerdes, Algeria, with a degree in electrical engineering in 1989, and a master's degree in microelectronics from the same institute in 1993. He then received a DSc in microelectronics and device physics from Mouloud Mammeri University in Tizi-Ouzou, Algeria. He has joined Mouloud Mammeri University in Tizi-Ouzou, Algeria in 1993. And he is a full professor and director of research at the present time. He is a member of the Board of Directors of the African Society of Physics and of the National Network of Microelectronics and Nanotechnologies, as well as a senior member of ICTP-UNESCO-IAEA, Trieste, Italy. He has supervised many doctoral theses in his research interests, including nanoelectronics, MOS systems and microelectronics.

Ahcene Lakhlef He received a degree in electronics engineering in 1988 and a master's degree in microelectronics in 1994 from the University of Sciences and Technology of Sétif, Algeria. He received his DSc degree from Mouloud Mammeri University, Tizi-Ouzou, Algeria, in microelectronics and device physics. He is currently a researcher for DSC at Mouloud Mammeri University, Tizi-Ouzou, Algeria. His research priorities include reliability, $\mathrm{C}(\mathrm{V})$ and $\mathrm{I}(\mathrm{V})$ semiconductor device technological characterization. 